\title{
ARE ANTIBIOTICS REQUIRED ROUTINELY DURING IMPLANT SURGERY? A CASE REPORT AND REVIEW
}

Jaibin George, Saumiya Gopal, ${ }^{2}$ Febel Huda, ${ }^{3}$ Niti Thomas.

1. Oral Implantologist, Cochin Implant Centre, Kalamaserry, Ernakulam

2. Periodontist, Cochin Implant Centre, Kalamaserry, Ernakulam

3. Prosthdontist, Cochin Implant Centre, Kalamaserry, Ernakulam

4. General Dentist, Cochin Implant Centre, Kalamaserry, Ernakulam

\section{Corresponding author:}

Dr. Saumiya Gopal,

Periodontist,

Cochin Implant Centre,

Kalamaserry, Ernakulam,

Phone Number - +8921032016,

E-mail ID : saumiyagopal@gmail.com

Submitted Date :10-01-2020 Accepted Date : 16-01-2020

\begin{abstract}
There has been a surge in the placement of dental implants as a treatment of missing teeth and it is also observed that antibiotics are being prescribed routinely with every implant case, irrespective of systemic status of the patient. In this article we discuss about a case in which multiple implants were placed into periodontally infected sites without antibiotics and has survived more than 6 years without any complications. This paper also reviews the literature regarding the scientific basis regarding using or not using antibiotics during implant surgery as well as the placement of implants into infected sites
\end{abstract}




\section{INTRODUCTION}

Dental implants have become widely the treatment of choice for replacement of missing teeth and invariably dentists prescribe antibiotics in every implant case to prevent infection. Although the choice to prescribe antibiotics should be case based, a study by Palmer et al in 2000 revealed that $40 \%$ of dentists would prescribe antibiotics to patients without any medical history as a contingency for infection. If infection does occur, the chances of implant failure are high. Though a number of factors can ultimately lead to implant failure, most practitioners are extra cautious regarding infection?

There are several adverse effects associated with antibiotics usage, ranging from diarrhea to life threatening allergic reactions. The biggest concern associated with the widespread use of antibiotics is the evolution of antibiotic resistant bacteria, along with the routine use of antibiotics may lead to laxsurgical techniques and actually increase the rate of complications. ${ }^{5,6}$ While prescribing antibiotics dentists should weigh the risk-benefit ratio.

Immediate implant placement into fresh extraction sockets has shown to have predictable outcome but placement into infected sites is considered relative contraindication. This paper presents multiple implants placed into infected sites without antibiotics and reviews the need of antibiotics based on evidence.

\section{CASE REPORT}

A 37 years old female was referred to Cochin Implant Centre for implant placement. She complained of mobile teeth with pus discharge. On examination it was found that she had generalized grade III and grade II mobility of teeth, generalized suppuration and a diagnosis of chronic generalized periodontitis was given. (Figure 1,2,3)

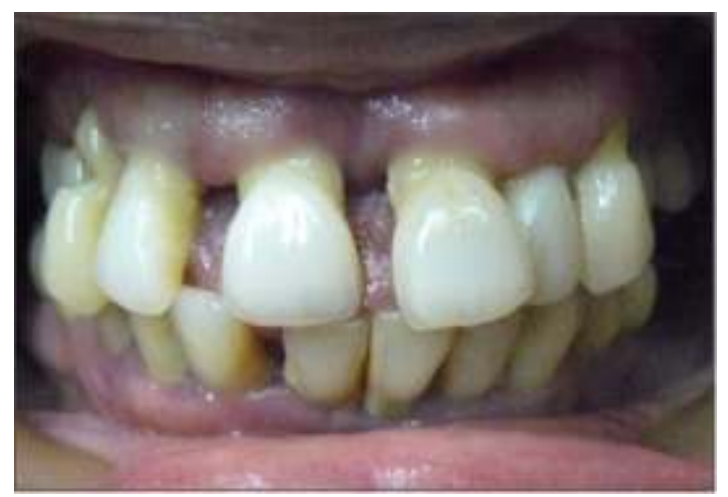

Fig 1: Pre-operative intra oral frontal view

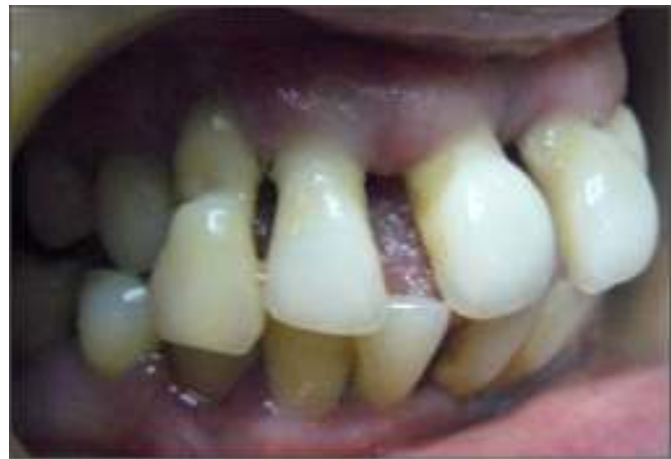

Fig 2: Intra oral lateral view

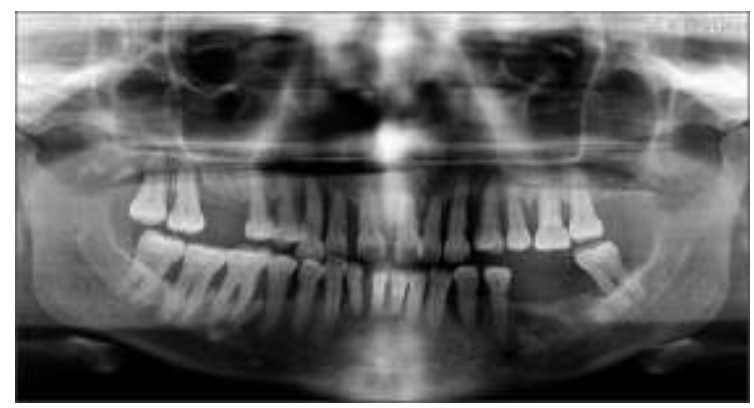

Fig 3: Pre-operative X Ray

Patient had no systemic diseases or drug allergies. She was posted for total extraction and immediate placement of implants but she informed at the beginning itself that her religious beliefs did not allow her to take any medications. She agreed for local anesthesia (LA) but refused to take even pain killers.

Extraction of $11,12,13,14,15,21,22,23,24,25,26$, $31,32,33,34,35,36,41,42,43,44,45$ and 46 was doneunder LA and twelve ADINToureg regular platform implants were placed. (fig 4,5)

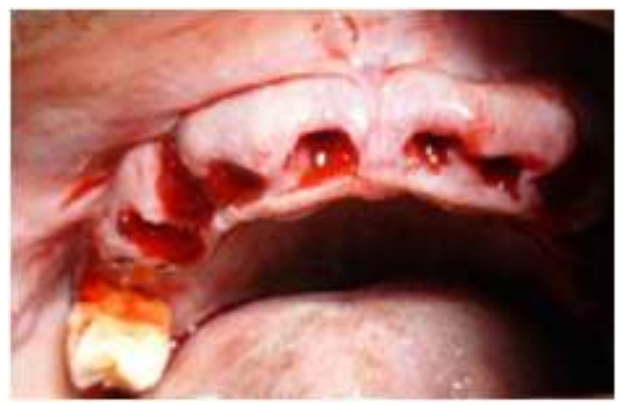

Fig 4: Immediately After Extraction Upper Arch

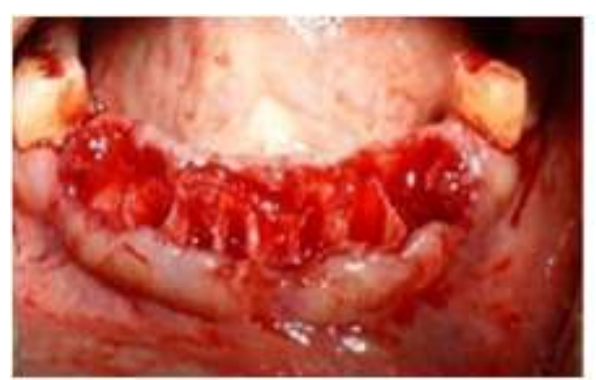

Fig 5: Immediately after extraction lower arch 
Six implants were placed in the upper arch with 6 in the lower. (fig 6)

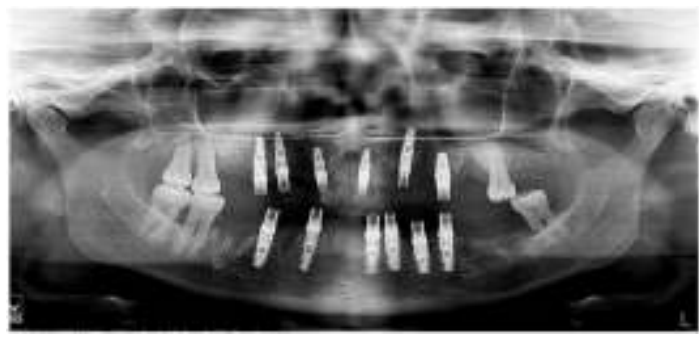

Fig 6: Immediate Post-op Opg

No biomaterials were used. All implants had more than $35 \mathrm{~N}$ primary stability. Titanium standard abutments were attached and abutment level impressions were made and a heat cure acrylic provisionals were delivered the next day. Patient was on provisionals were 3 months and after that provisionals were removed and implant level impressions were made. A screw retained hybrid prosthesis was delivered. (fig 7)

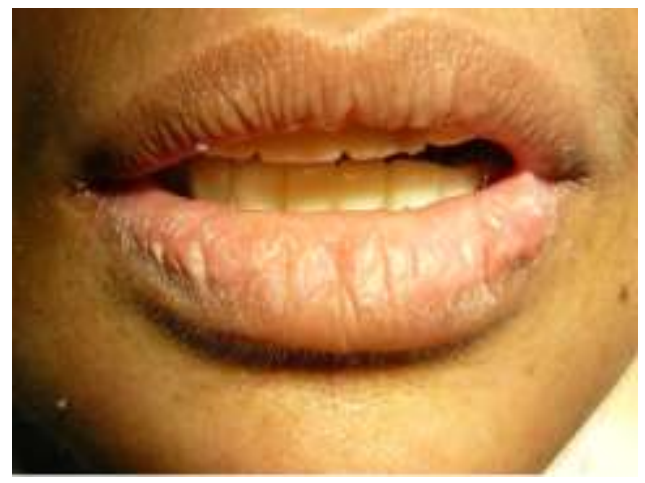

Fig 7: Post-op

On regular follow ups, the patient presented with no complications and crestal bone levels were maintained. (fig 8)

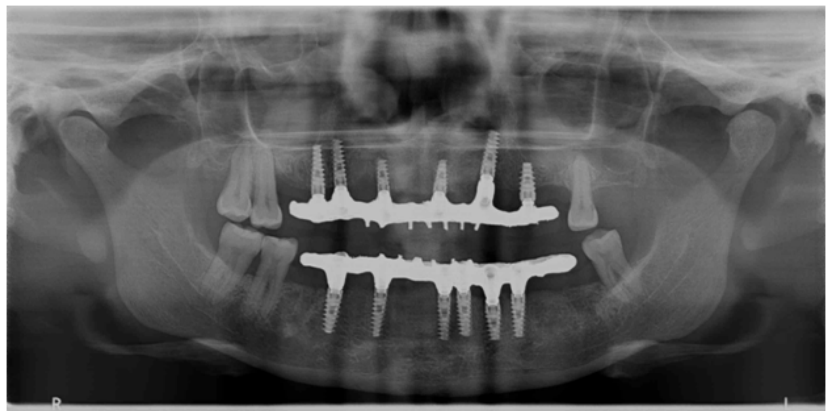

Fig 8: Two Years Post-op Opg
The teeth which were not extracted showed to improve in periodontal status over the past years. The patient has been on regular checkup for the past 6 years.(fig 9 )

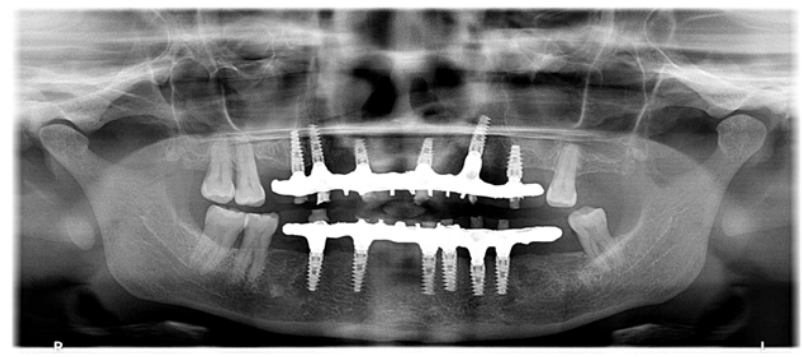

Fig 9: Six Years Post-op Opg

\section{DISCUSSION}

With antibiotics being used widely and without evidence, the need to check our routine practice of prescribing antibiotics with every implant case is important.The literature review shows that there are equal number of studies supporting the usage of antibiotic prophylaxis and those contradicting the use of antibiotics. But many articles did not compare between no antibiotics, preop, post-op and both pre and post-op? One of the common feeling amongst most of the papers was that antibiotics are being overused and that the authors requested that all practitioners assess each patient individually in the hopes of reducing the amount of prescribed antibiotics.

Ahmad et al found that of the 11406 implants they reviewed, cases with no antibiotics had a $92 \%$ success rate, cases with pre-op antibiotic alone had a $96 \%$ success rate, cases with post-op antibiotic alone had a $97 \%$ success rate and cases with both pre and post-op antibiotic had a success rate of $96 \%$. Thus, the results from this literature review show a $>90 \%$ success rate when antibiotics are used compared to when theyare not used. Thus, no benefit is seen from the use of antibiotic prophylaxisin low and moderate risk dental implant patients.

Gynther et al retrospectively compared the outcomes of dental implant treatment with and without antibiotic prophylaxis. Two groups of patients with edentulous or partially edentulous maxillas or mandibles (or both) were treated with dental implants. One group, consisting of 147 patients (790 implants), was given prophylaxis with oral phenoxymethyl penicillin; $1 \mathrm{~g}$ of antibiotic was 
administered 1 hour preoperatively, and $1 \mathrm{~g}$ was administered every 8 hours for 10 days postoperatively. The other group, consisting of 132 patients (664 implants) was not given any antibiotics preoperatively or postoperatively. There were no significant differences with respect to early and late postoperative infections or with respect to implant survival between the two groups. They concluded that antibiotic prophylaxis for routine dental implant surgery offers no advantage for the patient.

A Cochrane review by Esposito et al assessed the beneficial or harmful effects of systemic prophylactic antibiotics at dental implant placement versus no antibiotic/placebo administration and, if antibiotics are of benefit, to find which type, dosage and duration is the most effective. ${ }^{9}$ They concluded that some evidence exist suggesting that $2 \mathrm{~g}$ of amoxicillin given orally $1 \mathrm{~h}$ preoperatively significantly reduce failures of dental implants placed in ordinary conditions. No significant adverse events were reported. They propounded that it might be sensible to suggest the use of a single dose of $2 \mathrm{~g}$ prophylactic amoxicillin prior to dental implant placement. It is still unknown whether post-operative antibiotics are beneficial, and which is the most effective antibiotic.

Sharaf et al. in 2011 conducted an evidencebased review to evaluate the implant failure in those who were given perioperative antibiotic therapy as compared with those who did not take any antibiotics..$^{10}$ They concluded that a single dose of antibiotic therapy preoperatively may slightly decrease the failure rate of dental implants.

Implants were placed into infected sites in the present case and it is often avoided or a course of antibiotics are prescribed before the extraction of infected teeth. There is limited evidence suggesting that there are comparable results between immediate implant placement in infected sites versus non infected sites. ${ }^{11}$ The systematic review concluded that immediate implant placement into infected sites tends to be a safe choice and carry a clinically acceptable prognosis, provided that the treatment plan has been carefully considered. The authors suggested the following prerequisites to be considered: proper prescription of systemic antibiotics, use of an oral rinse before and after surgery, thorough debridement of the extraction sockets, and effective load management of interim and definitive restorations.
Waasdorp et al in their systematic review concluded that evidence suggests implants can be placed into sites with periodontal or periapical infections. The sites must be thoroughly debrided prior to placement. Guided bone regeneration is usually performed to fill the bone-implant gap and/or socket deficiencies. Although controversial, systemic antibiotics should be used until further controlled trials prove otherwise.

Another systematic review and metaanalysis by Chen et al in 2018 analyzed the treatment outcomes of immediate implant placement into extraction sockets with or without infection of periodontal or periapical origin in the esthetic zone and to provide treatment protocols based on current studies..$^{12}$ Their meta-analysis showed that immediate implant placement into infected sites and non-infected sites in esthetic zone had similar survival rates, bone level changes, and gingiva level changes.

Although literature supports limited use of antibiotics, clinicians are over prescribing them. The dental professionals should diligently administer antibiotics only when needed, thus avoiding unnecessary allergic reactions whenever possible. The most common adverse effects of antibiotics are direct toxicity, hypersensitivity reactions, and the short or long term development of resistant microorganisms. Direct toxicity includes gastrointestinal (nausea, vomiting, diarrhea, and abdominal pain), hematological concerns (neutropenia, thrombocytopenia, and hemolysis), alterations in the body's normal flora leading to candidal infections or pseudomembranous colitis, nephrotoxicity (proteinuria or renal failure), neuropathy (nerve dysfunction or peripheral neuropathy), alterations in drug interactions, and finally hepatobiliary (jaundice or hepatitis). ${ }^{13}$

The adverse effects associated with use of antibiotic usage must be assessed in comparison to the costs and morbidity related to treating infective endocarditis or infected prosthetic materials. If the risk-benefit and cost-benefit ratios are thoroughly evaluated, it becomes clear that if there are specific therapeutic indications based on sound physiologic, anatomic and scientific evidence, then antibiotic prophylactic therapy may be justified. Clinicians should diligently prescribe antibiotics after taking every factor into consideration. 


\section{CONCLUSION}

The routine use of systemic antibiotics in dental implant placement is not supported in literature. Antibiotic usage does not appear to influence the early incidence of prosthesis failure, implant failure, adverse events, or post operative complications. Maintaining an aseptic field while surgery and following all surgical protocols will help in reducing the infection and implant failure that clinicians fear.

\section{REFERENCES}

1. Palmer NO, Martin MV, Pealing R, Ireland RS. An analysis of antibiotic prescriptions from general dental practitioners in England. $\mathrm{J}$ Antimicrob Chemother 2000;46(6):10331035.

2. Lawler B, Sambrook PJ, Goss AN. Antibiotic prophylaxis for dentoalveolar surgery: is it indicated? Aust Dent J. 2005;50(4 Suppl 2):S54-59.

3. Beikler T, Flemmig TF. Implants in the medically compromised patient. Crit Rev Oral Biol Med. 2003;14(4):305-316

4. Ziment I. Complications of antibiotic therapy. Calif Med. 1972;117(5):24-48.

5. Esposito M, Coulthard P, Oliver R, Thomsen P, Worthington HV. Antibiotics to prevent complications following dental implant treatment. Cochrane Database Syst Rev. 2003(3):CD004152.

6. Mazzocchi A, Passi L, Moretti R. Retrospective analysis of 736 implants inserted without antibiotic therapy. J Oral Maxillofac Surg. 2007;65(11):2321-2323.

7. Nabeel Ahmad, Najeeb Saad. Effects of Antibiotics on Dental Implants: A Review. J Clin Med Res. 2012;4(1):1-6

8. Gynther GW, Köndell PA, Moberg LE, Heimdahl A. Dental implant installation without antibiotic prophylaxis . Oral Surg Oral Med Oral Pathol Oral Radiol Endod. 1998 May;85(5):509-11.

9. Esposito M, Grusovin MG, Loli V, Coulthard $\mathrm{P}$, Worthington HV. Does antibiotic prophylaxis at implant placement decrease early implant failures? A Cochrane systematic review. Eur J Oral Implantol. 2010 Summer;3(2):101-10.

10. Sharaf B, Jandali-Rifai M, Susarla SM, Dodson TB. Do perioperative antibiotics decrease implant failure? J Oral Maxillofac Surg. 2011;69:2345-50.

11. Ahmed Yaseen Alqutaibi. Limited evidence suggests that the immediate placement of dental implants into infected sites versus noninfected sites in the esthetic zone show comparable clinical results. J Evid Base Dent Pract 2019: [180-182].

12. Chen H, Zhang G, Weigl P, Gu X. Immediate placement of dental implants into infected versus noninfected sites in the esthetic zone: A systematic review and meta-analysis.. J Prosthet Dent 2018; 120(5):658-71.

13. Ziment I. Complications of antibiotic therapy. Calif Med. 1972;117(5):24-48. 